\title{
VLBI WITH TDRSS
}

\author{
R. P. LINFIELD \\ Jet Propulsion Laboratory 4800 Oak Grove Dr., Pasadena, CA 91109, USA
}

\begin{abstract}
VLBI observations using a satellite in earth orbit and ground antennas in Japan and Australia were conducted in 1986, 1987, and 1988. Sources were detected on space-ground baselines at both observing frequencies: 2.3 and $15 \mathrm{GHz}$. The coherence on space-ground baselines for $340 \mathrm{~s}$ was $90 \%$ at $2.3 \mathrm{GHz}$ and $76 \%$ at $15 \mathrm{GHz}$. Brightness temperatures in the range $1-4 \times 10^{12} \mathrm{~K}$ were measured for 10 sources at $2.3 \mathrm{GHz}$ and 6 sources at $15 \mathrm{GHz}$.
\end{abstract}

\section{Introduction}

The Tracking and Data Relay Satellite System (TDRSS) consists of satellites in geostationary orbit, designed to relay data between a ground station in White Sands, New Mexico, USA (WSGT) and satellites in low earth orbit (e.g. the Hubble Space Telescope, the NASA space shuttle). Each satellite has two $4.9 \mathrm{~m}$ diameter antennas, equipped with 2.3 and $15 \mathrm{GHz}$ receivers (and transmitters), with bandwidths of 16 and $256 \mathrm{MHz}$, respectively. A tone from a ground frequency standard is broadcast from WSGT and used to phase-lock all on-board oscillators.

TDRSS satellites appear to be the most suitable existing satellites for space VLBI, due to their local oscillator scheme, high-gain antennas, and large received bandwidths. A TDRSS satellite was used for three space VLBI experiments: July/Aug. 1986 (2.3 GHz only), Jan. 1987 (2.3 GHz only), and Feb./Mar. 1988 ( 2.3 and $15 \mathrm{GHz}$ ). The purpose was to test several technical concepts peculiar to space VLBI, and to perform a survey of the brightest sources to measure their size distribution.

\section{Observations}

At the time of these experiments, only one TDRSS satellite: TDRSA, was in orbit, above $41^{\circ} \mathrm{W}$. longitude. It was constrained to look back towards the earth: within $31^{\circ}$ of the nadir in declination, and within $22^{\circ}$ in hour angle (Figure 1 ). The ground radio telescopes used in these experiments therefore needed to be located approximately $180^{\circ}$ in longitude away from the sub-earth point of TDRSA. Antennas in Japan (the Usuda $64 \mathrm{~m}$ antenna, NRO $45 \mathrm{~m}$, and Kashima $26 \mathrm{~m}$ ) and Australia (64/70 $\mathrm{m}$ and $34 \mathrm{~m}$ antennas of the NASA Deep Space Network in Tidbinbilla).

The Mk III recording system was used for all 3 experiments. In the first two, a $14 \mathrm{MHz}$ bandwidth of $2.3 \mathrm{GHz}$ data was recorded. In the third experiment, the Mk III double speed mode was used to record $88 \mathrm{MHz}$ bandwidth at $15 \mathrm{GHz}$, and $12 \mathrm{MHz}$ bandwidth at $2.3 \mathrm{GHz}$. Data from TDRSA were broadcast to the ground (WSGT), where they were digitized and recorded. Details of the experimental procedure are given in references 1 and 2. The data correlation was performed at

(C) 1990 Kluwer Academic Publishers. Printed in The Netherlands. 


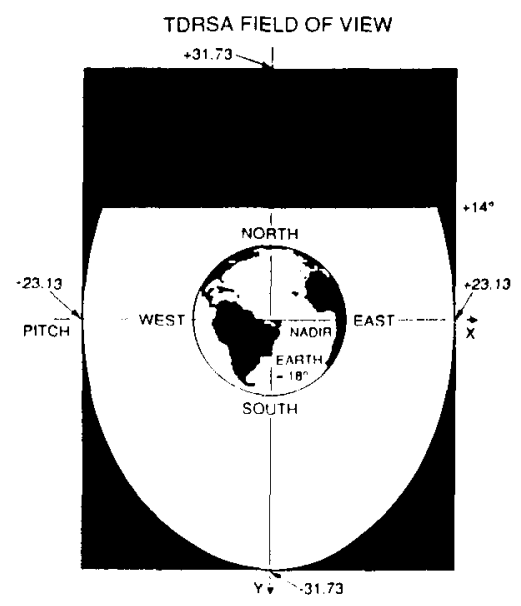

Fig. 1. TDRSS field of view, drawn to scale.

Haystack Observatory, using the Mk IIIA correlator. Software modifications were needed to allow data from an orbiting antenna to be correlated.

\section{Coherence}

The coherence measured in the third experiment is shown in Figure 2. Both the shape of the coherence curves (substantial loss over short integration times) and the frequency dependence (the coherence degrades quite slowly with increasing frequency) suggest that neither the ground frequency standard nor orbit determination errors are the primary sources of coherence loss. A more likely cause is frequency flicker noise generated in the on-board local oscillator chain (which was not designed to do VLBI).

\section{Source Visibilities and Brightness Temperatures}

In the second experiment, where the majority of the $2.3 \mathrm{GHz}$ data were obtained, 23 out of 24 sources were detected on TDRSA-ground baselines. The longest projected baseline length (limited by TDRSA pointing constraints) was 2.15 earth diameters $\left(D_{\oplus}\right)$. Three sources were detected on baselines longer than $2.0 D_{\oplus}$. The most compact source was $1519-273$, with a visibility of 0.66 on a $2.02 D_{\oplus}$ baseline. Sufficient data were obtained on 14 sources to determine brightness temperatures. 10 of those sources had brightness temperatures in the range $1-4 \times 10^{12} \mathrm{~K}$ (Figure 3 , reference 3 ), exceeding the $1 \times 10^{12} \mathrm{~K}$ Inverse Compton limit.

At $15 \mathrm{GHz}$, the detection rate on TDRSA-ground baselines was lower: 11 of 22 sources. However, the sensitivity of the interferometer was much poorer than at $2.3 \mathrm{GHz}$. The observed brightness distributions of source visibilities and brightness temperatures were similar at the two frequencies. 


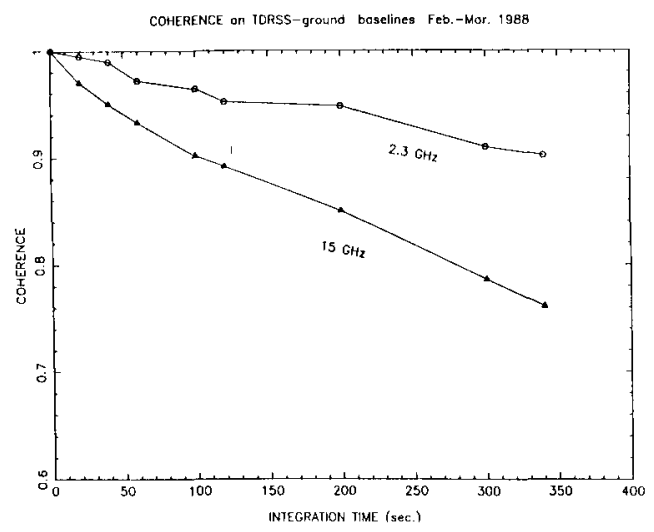

Fig. 2. Coherence values for TDRSS baselines.

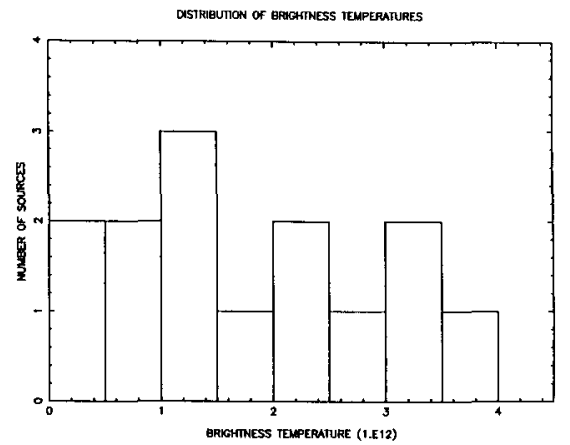

Fig. 3. Histogram of $2.3 \mathrm{GHz}$ brightness temperatures from Jan. 1987 experiment.

\section{Discussion}

These experiments demonstrated that space VLBI observations can be successfully performed, even with a spacecraft not designed for VLBI. With careful designs, VSOP and Radioastron should be able to achieve excellent coherence.

The measured distribution of sources sizes and brightness temperatures demonstrates that baselines of $1-3 D_{\oplus}$ will be very useful for studying the structure of bright, compact radio sources. The existence of correlated flux on baselines longer than $3 D_{\oplus}$ is still an open question, awaiting Radioastron observations for its answer.

The TDRSS experiments involved a large multinational effort led by G. Levy of JPL, with major roles from groups in Japan and Australia. 


\section{References}

Levy, G. S. et al.: 1989, Ap. J. 336, 1098

Linfield, R. P. et al.: 1990, Ap. J. , (in press)

Linfield, R. P. et al.: 1989, Ap. J. 336, 1105 